\title{
STUDY ON OPTIMIZATION OF STIR HEAD FOR FSW BASED ON GENETIC ALGORITHM
}

\author{
Zhaoxin Meng ${ }^{1}$, Hui Chen ${ }^{1}$, Xihua Yue ${ }^{2}$ \\ ${ }^{\prime}$ School of Mechatronics Engineering, ${ }^{2}$ College of Foreign Languages, Northeast Forestry \\ University, China; Email:mzhxmail@I63.com.
}

\begin{abstract}
The shape selection of stir head is processed to Friction Stir Welding (FSW), and the mathematical model and constraints for the optimization of stir head are established, what is more, the multi-target optimization algorithm of stir head dimension based on genetic algorithm is presented. Utilizing this algorithm, an example of optimization is analyzed, and its optimized finite element modeling is given, at last the reasonable dimension parameters are obtained.
\end{abstract}

Key words: Friction Stir Welding (FSW), Stir head, Optimization, Genetic algorithm

\section{INTRODUCTION}

Friction Stir Welding (FSW), which is a new solid phase welding process, has been largely used in aluminum alloys welding ${ }^{1}$. To date, FSW has attracted considerable interest because of its better weld ability of aluminum alloy weld joint, and considerable research on FSW has been carried out. However, research is lacking on the optimization procedure of FSW, it is well known that stir head is a critical component of the friction welding machine, and the quality of the weld joint is dependent on its property. So, this situation has led to the study on optimization procedure of stir head for FSW in order to be superior in property to any other stir head.

Please use the following format when citing this chapter:

Meng, Zhaoxin, Chen, Hui, Yue, Xihua, 2006, in International Federation for Information Processing (IFIP), Volume 207, Knowledge Enterprise: Intelligent Strategies In Product Design, Manufacturing, and Management, eds. K. Wang, Kovacs G., Wozny M., Fang M., (Boston: Springer), pp. 483-491. 


\section{SELECTION OF STIR HEAD SHAPE}

\subsection{The shape of shaft shoulder}

The function of shaft shoulder is to provide friction contact points and sealed welding condition so that plastic metal cannot be extruded through it ${ }^{1}$. In the middle of the shaft shoulder end, the general shape is concave, especially the juncture of the stir needle and the shaft shoulder. Therefore, the general shape of shaft shoulder, the end of which is concave, has been selected ${ }^{2,3}$.

\subsection{The shape of stir needle}

With a cone screw stir head, it has been proved that weld joint has higher tensile strength. And what is more, with a tapered thread stir head, tensile strength is more stable with the welding speed changing. Material in heat-affected zone of cone screw is harder, and the hardness of onward side of cone screw weld joint is clearly larger than usual.

In the process of welding, plastic material receives a kind of shear force from screw thread, and receives a kind of downward pressure from conical surface at the same time, since tapered thread has cone angle. The affection of these two forces can make the crystal grains in the heat-affected zone elongated, where slender grains that should be parallel with conical surface come into being. The temperature duration of receding side of weld joint is slightly longer, so the hardness of receding side is lower with the larger size of grains ${ }^{4}$.

With a tapered thread stir head, weld joint has higher tensile strength. And what is more, with a tapered thread stir head, tensile strength is more stable with the welding speed changing, therefore, a tapered thread stir head has been selected in the research. 

Algorithm

\section{MATHEMATICAL MODEL FOR THE OPTIMIZATION OF STIR HEAD DIMENSION}

\subsection{Mathematical model for the optimization of stir head dimension}

It is supposed that $R_{0}$ is the middle diameter of stir needle, $L$ is the length, $R_{2}$ is the lower extreme diameter of it, and $R_{1}$ is the upper extreme diameter of it, and $R_{2} \leq R_{1}$. In the process of welding, we suppose that the welding speed is $v$, the cone angle of stir needle is $\alpha$, and rotating speed is $\omega$.

In the process of welding, the material ahead of stir needle has been under high plastic state, which brings to bear pressure intensity to stir needle, $P$ is referred to the pressure intensity.

As the stir needle moves forward, welding materials engender plastic deformation in response to mechanical forces, the volume of which is $\Delta S_{u}$ when stir needle has moved $\Delta S$ after $\Delta t$, and the volume of the plastic metal that stir needle extrude is $\Delta J$, from the given information, we find that the thread any infinitesimal rotates is $\Delta t \cdot \omega \cdot r$, using this equation, the generated heat quantity is calculated as:

$$
\Delta Q=F \cdot \Delta t \cdot \omega \cdot r=\frac{P_{s} \cdot f \cdot r \cdot \Delta r \cdot \Delta \theta}{\sin \alpha} \cdot \Delta t \cdot \omega \cdot r
$$

After $\Delta t$, we find that the friction heat generated from the front of the stir needle is:

$$
\Delta Q_{Z}=\int_{R 2}^{R_{1}} \int_{0}^{\pi} \frac{P_{s} \cdot f \cdot r^{2} \cdot \omega}{\sin \alpha} d r \cdot d \theta=\frac{\pi \cdot P_{s} \cdot f \cdot \omega \cdot \Delta t}{3 \sin \alpha}\left(R_{1}^{3}-R_{2}^{3}\right)
$$

and the heat comes from shaft shoulder is calculated as:

$$
\Delta Q_{J}=\frac{4 \pi \omega \cdot f \cdot F \cdot\left(R^{3}-R_{0}^{3}\right)}{3 \cdot R^{3}} \Delta t
$$


where $F$ is the axial pressure from shaft shoulder and $R$ is the diameter of shaft shoulder.

Thus, solving for the heat output coefficient $\Delta Q / \Delta t$ and substituting the values gives:

$$
\frac{\Delta Q}{\Delta t}=\frac{\Delta Q_{Z}+\Delta Q_{J}}{\Delta t}=\pi P_{s} \cdot f \cdot \omega \cdot\left(\frac{L^{3} \cdot \operatorname{tg} \alpha}{12 \cos \alpha}+\frac{R_{0}^{2} \cdot L}{\cos \alpha}\right)+\frac{4 \pi \omega \cdot f \cdot F \cdot\left(R^{3}-R_{0}^{3}\right)}{3 \cdot R^{3}}
$$

The parameters of the heat input coefficient with relation to the stir head are $L, R, R_{0}$ and $\alpha$.

Since $L$ is always thinner than welding material thickness, it is not likely to change. It is appreciable that $R$ and $R_{0}$ should be optimized, for they are the decisive parameters of stir head and the heat output coefficient of stir needle. From the given numerical model, we find that the value of the cone angle of stir needle $\alpha$ can influent the heat input of stir needle, in practice, too large $\alpha$ will not be considered because of its decreasing the capacity of undergoing impact forces, and too small $\alpha$ will also not be considered because the proximate cylindrical shape of stir head is not ideal. According to the above analysis, it is necessary to optimize the diameter of shaft shoulder and the diameter of stir needle in this study.

In the process of FSW, the strength of weld joint is connect with the shape of the stir head, the shape parameters of this frustum stir head are considered to be the diameter of shaft shoulder and the diameter of stir needle. It is important recognize, however, the relation between them is not functional, but statistical. In this study, the optimization of stir head for FSW has considered the diameter of shaft shoulder and the diameter of stir needle as independent variable, which are selected within numeral value. At this point, in establishing mathematical model for the optimization of stir head, the diameter of shaft shoulder $R$ and the diameter of stir needle $R_{0}$ should be restricted: $R=17 \sim 27 \mathrm{~mm}, R_{0}=3 \sim 5 \mathrm{~mm}$.

\subsection{Mathematical model for the heat input coefficient of stir head}

We have known the heat output coefficient $\Delta Q / \Delta t$ is calculated as:

$$
\frac{\Delta Q}{\Delta t}=\frac{\Delta Q_{Z}+\Delta Q_{J}}{\Delta t}=\pi P_{s} \cdot f \cdot \omega \cdot\left(\frac{L^{3} \cdot \operatorname{tg} \alpha}{12 \cos \alpha}+\frac{R_{0}^{2} \cdot L}{\cos \alpha}\right)+\frac{4 \pi \omega \cdot f \cdot F \cdot\left(R^{3}-R_{0}^{3}\right)}{3 \cdot R^{3}}
$$



Algorithm

The parameters of the heat input coefficient with relation to the stir head are $L, R, R_{0}$ and $\alpha$.

\section{DESIGN OF THE ALGORITHM FOR THE OPTIMIZATION OF STIR HEAD DIMENSION}

\subsection{Multi-target optimization based on genetic algorithm}

The most typic method to solve problems of multi-target optimization is to integrate several objective functions in the way of weighted average so that one objective function, which may be solved easily, can be achieved. However, the achieved objective function has no definite physical meaning, though separate objective functions have different physical meaning. Moreover, since order of magnitude of different objective functions should be different, the weighted value cannot be used to substitute the proportion of the integrated objective function. And it is a fact that the weighted value, which is selected in term of the designer's experience, will tamper with the objectivity.

Considering the given reason, a new multi-target optimization with constraint based on genetic algorithm is utilized for the optimization of stir head dimension.

When genetic algorithm is selected to optimize multi-target function, this study will not optimize the integrated objective function only, but all objective functions at the same time. A optimum solution to the multi-target optimization is on the lower left boundary of the doable range of values, therefore, if the lower left boundary of the doable range of values can be found, we can select the optimum solution directly according to the actual need.

\subsection{The operation process of algorithm}

There are several processes in the operation of algorithm. First of all, a certain number initial chromosomes are created at random, which make up of a population; then, a degree of adaptability function will confirm the degree of adaptability of each individual, which is to circumstance. The next thing to do is to select the chromosomes to obtain a new population, and this new population will perform chiasm operation and variation operation. The repeating selection operation, chiasm operation, and variation operation will be performed until the given iterations are fulfilled. Fig.1 shows the 
operation process of algorithm, according to the principium and process of genetic algorithm.

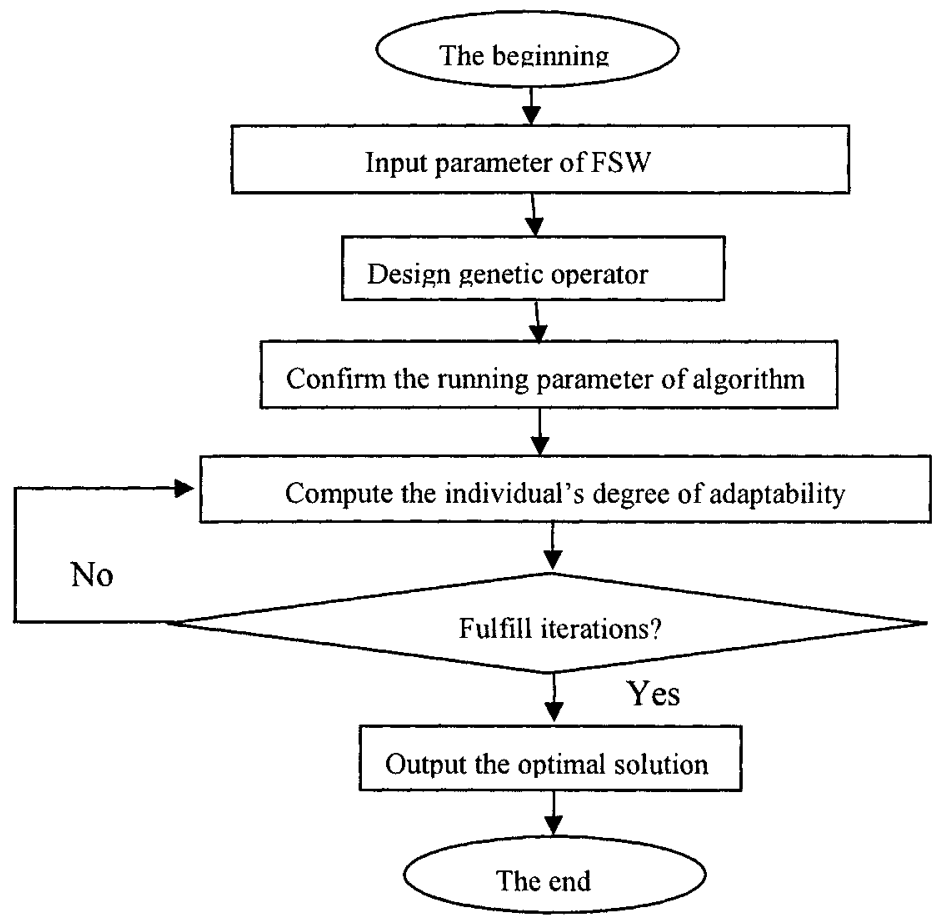

Figure 1. The operation process of algorithm for the optimization of stir head dimension

\subsection{The retention strategy of algorithm and the analysis of astringency}

The fundamental genetic algorithm can be described as an odd capital Markov chain, the reason is that the selected operation, chiasm operation, and variation operation should be performed autonomously and stochastically, new populations are connected with their father's population, but are not the former populations, that is, populations have no after working. And the transition probability between every two populations has nothing with the initial point of time. Therefore, the probability that the fundamental genetic algorithm can converge to the optimal solution is less than 1.

However, in theory, the genetic algorithm in this study must be ameliorated so that the genetic algorithm can converge to the optimal solution. So, optimal retention strategy is used in this study, which is helpful to converging the optimal solution, and its probability will be 1 . 


\section{CASE ANALYSIS}

\subsection{Computation of case}

EXAMPLE: Aluminum-lithium alloy is new material used in the modern aerospace field because of its excellent properties; however, it is always a difficulty to connect with each other. This FSW joining method can largely promote the joining property of Aluminum-Lithium alloy. Which kinds of shapes of stir head will be selected has been the key point of Friction Stir Welding, something in common is the concave shape in the middle of the shaft shoulder end, especially the juncture of the stir needle and the shaft shoulder, and the stir needle is frustum. So, a stir head for opposite joint of Aluminum-Lithium alloys with straight stitch, of which the serviceable thickness is $5 \mathrm{~mm}$, has been optimized as follows ${ }^{6}$.

This study threats the given mathematical model as the objective function, the value of the length of coding string: $I=50$, the value of the size of population: $M=80$, the value of the chiasm probability: $p=0.5$, and the terminate algebra: $T=50$.

After optimizing the stir head, the study has calculated: $R=13.4 \mathrm{~mm}$, $R_{0}=4.2 \mathrm{~mm}$. This optimized finite element modeling is given.Fig. 2 shows the optimized finite element modeling of stir head.

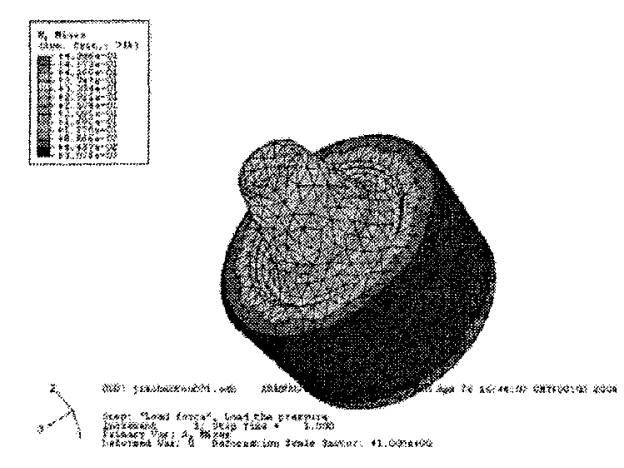

Figure 2. The optimized finite element modeling of stir head

\subsection{The mechanical property analysis of the weldment with optimal stir head}

The test result of weld strength has showed that the weld joint strength with cone screw, of which $R$ is $13.4 \mathrm{~mm}, R_{0}$ is $4.2 \mathrm{~mm}$, can reach to $370 \mathrm{MPa}$, 
the highest value of the strength, this is consonant with the result of the optimized mathematical model of stir head size. Fig. 3 has clearly showed that weld strength will go up with the growth of weld speed. It is delightful that this stir head has the highest strength in the experiment; moreover, it is most likely that the highest strength can be increased. Therefore, if the diameter of shaft shoulder $R$ is $13.4 \mathrm{~mm}$ and the diameter of stir needle $R_{0}$ is $4.2 \mathrm{~mm}$, the cone screw stir head can be considered as the optimal stir head.

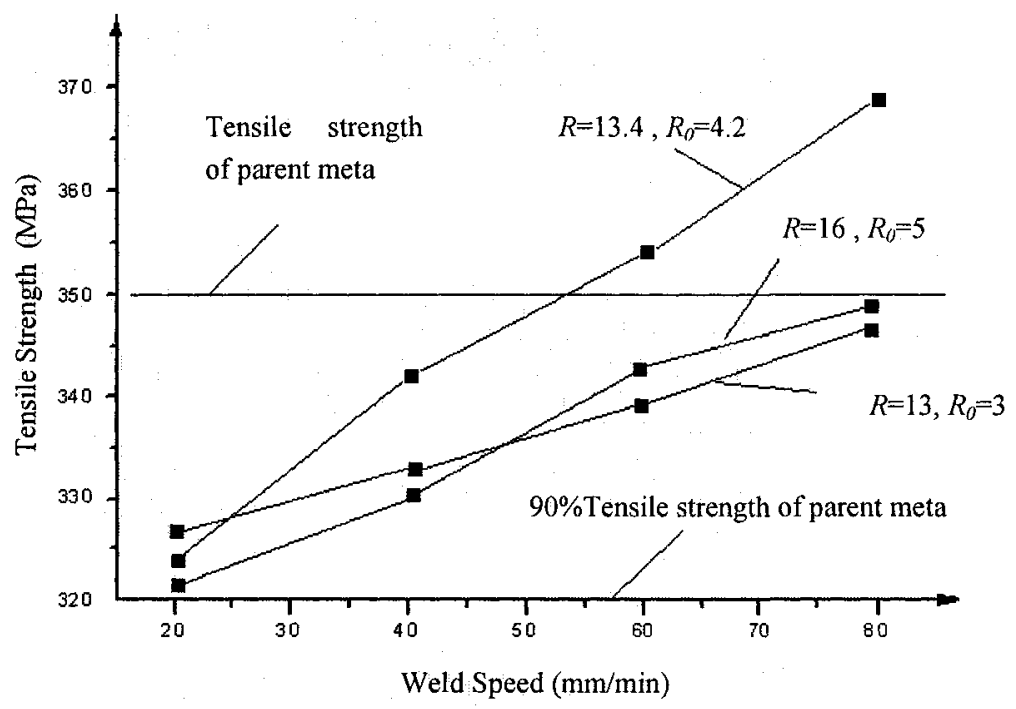

Figure 3. Effect of weld speed on the strength of the optimal stir head

\section{CONCLUSION}

- The cone screw stir head has been selected according to the analysis to its advantages.

- The mathematical model for the optimization of stir head is established, which has been treated as the optimized objective function.

- The optimization algorithm and its running parameters of the stir head dimension based on genetic algorithm are presented by analyzing the convergent of the algorithm.

- It has been proved that the stir head in this study is the optimal stir head after optimization. The character of genetic algorithm such as easy 
fulfilling, unrelated problems and overall convergent is making its practicability and application potential extensive.

- In the process of running, considering different processing conditions, different running parameters of algorithm can be selected, and more reasonable stir head can be obtained by several pilot calculations to the running parameters.

- The optimization of stir head dimension can be regarded as a problem of multi-target optimization, of which the way of weighted average has intrinsic limitation. A new multi-target optimization with constraint based on genetic algorithm can be utilized for the optimization of multitarget function, with the application expedient and rapid, and the optimized result can also reflect the internal relation between the objective functions.

\section{REFERENCES}

1. Lohwasser D. Application of friction stir welding for aircraft industry. The 2nd International Symposium on Friction Stir Welding.Sweden: Gothenburg, 2000

2. Thomas MW, Nicholas ED, Needham JC, etal. Friction stir welding. GB Patent Application, 9125978. 1991-12-08

3. Lohwasser D. Welding of airframe by friction stir welding. The 3rd International Symposium on Friction Stir Welding. Japan: Kobe, 2001

4. Talwar R, Bolser D, Lederich R, etal. Friction stir welding of air frame structures .The 2nd International Symposium on Friction Stir Welding. Sweden: Gothenburg, 2000

5. Marie F. Development of friction stir welding of 7000 series extrusions for central wing box application. The 3rd International Sym 2posiumon Friction Stir Welding. Japan: Kobe, 2001

6. Pedwell $\mathrm{R}$, Davis $\mathrm{H}$, Jefferson A. The application of friction stir welding to aircraft wing.The lst International Symposium on Fric2tion Stir Welding. USA: Thousand Oaks, California, 1999 